\title{
Novel patterns of progression upon immunotherapy in other thoracic malignancies and uncommon populations
}

\author{
Roberto Ferrara $^{1,2}$, Diego Signorelli ${ }^{3}$, Claudia Proto ${ }^{1}$, Arsela Prelaj ${ }^{1,4}$, Marina Chiara Garassino ${ }^{1}$, \\ Giuseppe Lo Russo ${ }^{1}$
}

${ }^{1}$ Department of Medical Oncology, Thoracic Oncology Unit, Fondazione IRCSS, Istituto Nazionale dei Tumori Milano, Milan, Italy; ${ }^{2}$ Department of Research, Molecular Immunology Unit, Fondazione IRCCS Istituto Nazionale dei Tumori di Milano, Milan, Italy; ${ }^{3}$ Department of Medical Oncology, Thoracic Oncology Unit, ASST Grande Ospedale Metropolitano Niguarda, Milan, Italy; ${ }^{4}$ Department of Electronics, Information, and Bioengineering, Polytechnic University of Milan, Milan, Italy

Contributions: (I) Conception and design: R Ferrara, G Lo Russo; (II) Administrative support: MC Garassino; (III) Provision of study materials or patients: R Ferrara; (IV) Collection and assembly of data: R Ferrara; (V) Data analysis and interpretation: R Ferrara, G Lo Russo; (VI) Manuscript writing: All authors; (VII) Final approval of manuscript: All authors.

Correspondence to: Roberto Ferrara, MD. Department of Medical Oncology, Thoracic Oncology Unit, Fondazione IRCSS, Istituto Nazionale dei Tumori Milano, Via Giacomo Venezian 1, 20133 Milan, Italy. Email: roberto.ferrara@istitutotumori.mi.it.

\begin{abstract}
In the immunotherapy era, considering the prolonged survival benefit and responses observed with immunecheckpoint inhibitors (ICI) in many cancer types, the identification of patients with rapid progression (PD) and deaths upon ICI has found some skepticism and resistance among the scientific community. Nevertheless, an acceleration of tumour during ICI, defined as hyperprogressive disease (HPD), has been recognized across different cancer types and evidence regarding rapid PDs and deaths are emerging in patients with malignant pleural mesothelioma (MPM), small cell lung cancer (SCLC) and thymic malignancies and in uncommon non-small cell lung cancer (NSCLC) populations. Of note, PD and early deaths (ED) rates upon single agent ICI were up to $60 \%$ and $30 \%$ in MPM and $70 \%$ and $38 \%$ in SCLC patients, respectively. Similarly, rapid PDs and deaths were observed in clinical trials and retrospective studies including patients with poor performance status (PS), HIV infection and rare NSCLC histologies. Atypical patterns of response, such as pseudoprogression (PsPD) may also occur in other thoracic malignancies (MPM) and in some uncommon populations (i.e., HIV patients), however probably at lower rate compared to HPD. The characterizations of HPD and PSPD mechanisms and the identification of common definition criteria are the next future challenges in this area of cancer research.
\end{abstract}

Keywords: Hyperprogressive disease (HPD); early deaths (ED); pseudoprogression (PsPD); other thoracic malignancies; uncommon populations

Submitted May 06, 2020. Accepted for publication Oct 21, 2020.

doi: $10.21037 /$ tlcr-20-636

View this article at: http://dx.doi.org/10.21037/tlcr-20-636

\section{Introduction}

Immunecheckpoint inhibitors (ICI) have radically changed the treatment scenario of many cancer types (1). Despite its unquestionable benefits, the advent of immunotherapy has also brought new challenges for medical oncologists regarding use of ICI combinations (2), role of predictive biomarkers (3) and of novel study methodologies (4).
In particular, the assessment of ICI efficacy in special subpopulations (5) and the characterization of atypical responses upon ICI (6) are clinical unmet needs. Although unconventional patterns of response [i.e., pseudoprogression (PsPD)] (7) or progression (PD) (i.e., hyperprogression) (8) have been described in ICI treatment advanced non-small cell lung cancer (NSCLC) patients, the mechanisms beyond them and the criteria to define these 
patterns have not been fully elucidated. Furthermore, few data are available for these novel patterns in patients with other thoracic malignancies and special subpopulations. The aim of this review is to discuss the current knowledge about hyperprogressive disease (HPD), PsPD occurring during ICI in patients with thoracic malignancies other than NSCLC and in uncommon populations such as elderly, patients with poor ECOG performance status (PS), autoimmune diseases, HIV infection or rare NSCLC histology subtypes. We will also provide an insight on the potential biological mechanisms associated with HPD and PsPD, focusing on some unsolved problems and areas of future research.

\section{Methods}

We performed a literature review regarding the association between the use of ICI and the occurrence of HPD or PsPD in patients with thoracic malignancies other than NSCLC and in uncommon populations. We searched digital databases including PubMed, the Cochrane Library and EMBASE. The survey was carried out using keywords such as "immunotherapy", "immune checkpoint inhibitors", "malignant pleural mesothelioma", "thymic carcinoma", "thymoma", "small-cell lung cancer", "antiPD-L1 antibody", "anti-PD-1 antibody", "anti-CTLA4 antibodies", "uncommon populations", "poor performance status", "HIV", "autoimmune diseases", "sarcomatoid lung cancer", "rare histology", "enteric lung adenocarcinoma", "hyperprogressive disease" "pseudoprogression", variously associated together. No language or period restrictions have been used. The great part of the studies was excluded according to the title or the abstract content. We have analyzed the full versions only for the most relevant papers. The reference lists of the most important studies were also evaluated.

\section{PD, HPD and early deaths (ED) in other thoracic malignancies and uncommon populations}

HPD, defined as a rapid disease growth during immunotherapy, has been reported in $3.8 \%$ (9) to $29.4 \%$ (10) of tumours and in $13.8 \%$ (11) to $37 \%$ (12) of advanced NSCLC patients treated with PD-1/PD-L1 inhibitors. In most of the studies including NSCLC patients, the median overall survival (OS) for patients with HPD ranged between 1.6-4.7 months $(12,13)$ and was lower compared to the median OS $(6.2-7.8$ months $)(11,14)$ of conventional disease
PD according to RECIST v1.1. No validated clinical or molecular predictors of HPD have been identified due to the heterogeneity and retrospective nature of the studies. Furthermore, there is no consensus on HPD definition and distinct criteria (i.e., one-dimensional, volumetric or clinical) have been proposed (15-17).

In the context of thoracic malignancies, most of the studies have described HPD in a dedicated NSCLC population and few data are available for patients with other thoracic malignancies. In fact, the existence of HPD was demonstrated only in retrospective studies because the definition criteria included the assessment of pre-ICI imaging (11-13) or the evaluation of metastatic spread upon ICI $(14,18)$ and both these data were not collected in clinical trials. Large retrospective real-world studies useful to assess HPD in thoracic malignancies other than NSCLC are currently missing because ICI are not standard treatments in these settings. However, rapid PDs and ED in the first 3 months of treatments have been reported in clinical trials testing ICI in patients with malignant pleural mesothelioma (MPM), small cell lung cancer (SCLC) or thymic malignancies, suggesting the existence of HPD across different thoracic cancers.

Similarly, HPD may occur in uncommon populations. In fact, HPD has been associated with aging (8) or with worsening of PS (14), in addition a remarkable increase in tumour burden has been reported in $\mathrm{HIV}^{+}$patients receiving $\mathrm{PD}-1 / \mathrm{PD}-\mathrm{L} 1$ inhibitors in clinical trials (19).

Although we reported that ED is not a surrogate of HPD in NSCLC patients, $55 \%$ of ED NSCLC patients experienced HPD according to tumour growth rate (TGR) variation (20). Therefore, in the absence of a proper evaluation of HPD in patients with MPM, SCLC and thymic malignancies enrolled in clinical trials, $\mathrm{PD}$ and $\mathrm{ED}$ rates may provide a rough estimate of HPD occurrence in these settings. A limitation to the use of ED would be the inclusion in this rate of toxic deaths from ICI, however considering that grade 5 events upon ICI are relatively rare, most of deaths labelled as ED can be considered due to radiological or clinical PD. In Table 1 we reported rates of PD, HPD, ED and PsPD in patients with thoracic malignanices other than NSCLC and in uncommon populations.

\section{PD, HPD and ED in MPM}

In pretreated MPM, tremelimumab, an anti-CTLA-4 monoclonal antibody, has been compared to placebo in 
Table 1 PD, HPD, ED and PsPD rates in other thoracic malignancies or uncommon populations

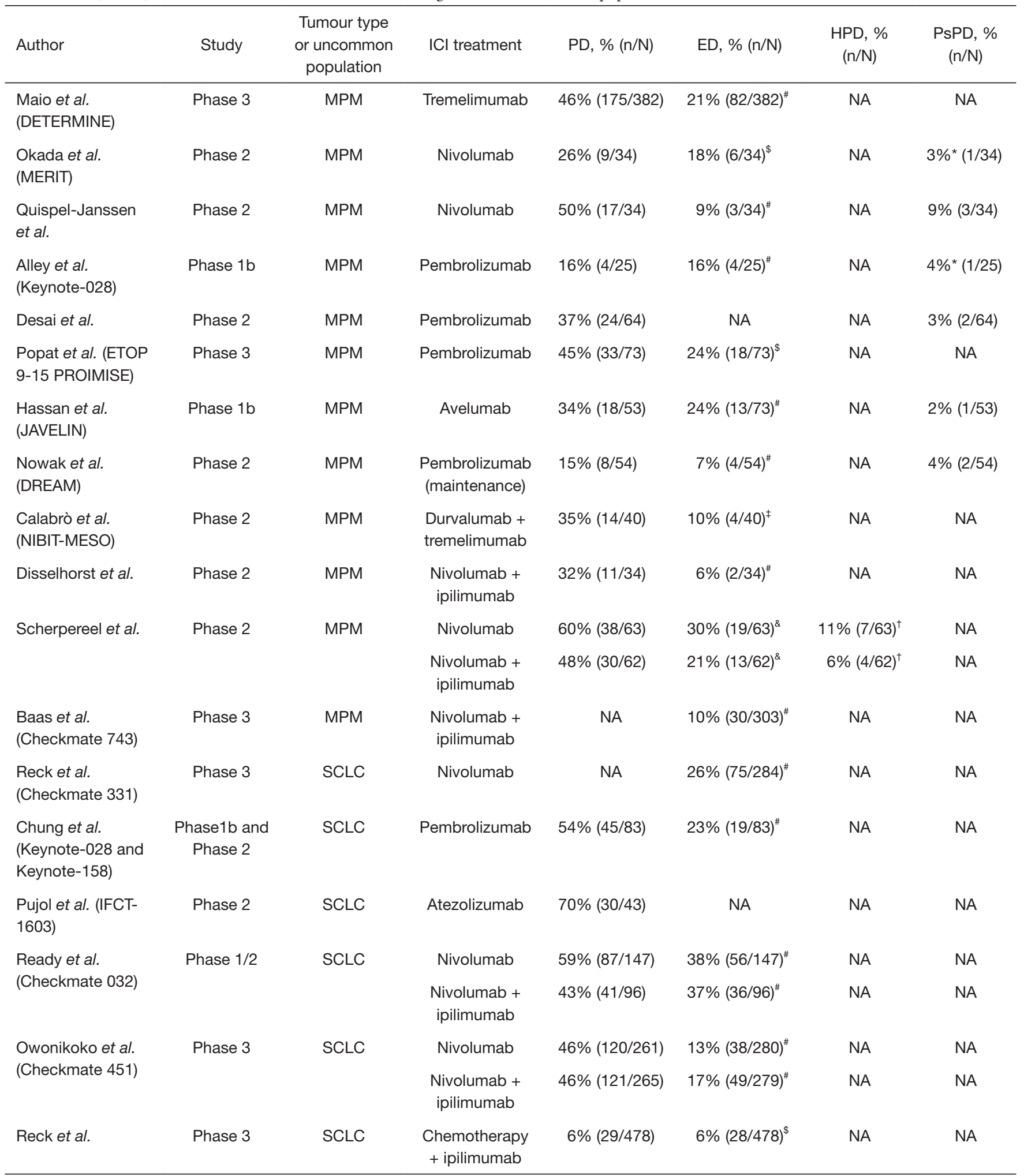

Table 1 (continued) 
Table 1 (continued)

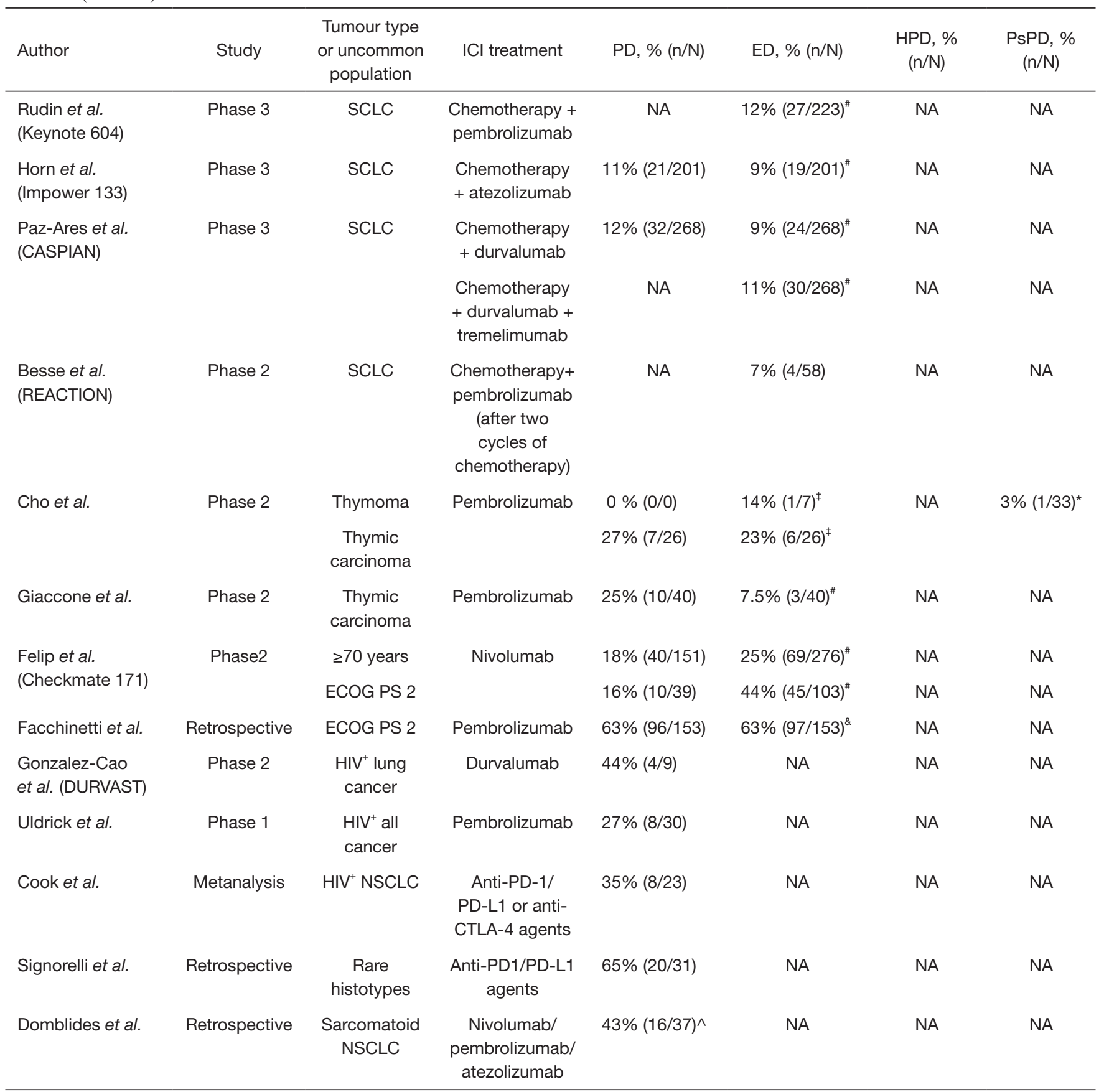

*, still on treatment beyond PD (not known the response beyond PD); , in the first 3 months from treatment initiation; ${ }^{\$}$, in the first 4 months from treatment initiation; ${ }^{*}$, in the first 5 months from treatment initiation; ${ }^{\ddagger}$, in the first 6 months from treatment initiation; ${ }^{\dagger}$, according to TGK ratio $\geq 2 ; \wedge, 32 \%$ of patients had a rapid PD. PD, progression; HPD, hyperprogressive disease; ED, early deaths; PsPD, pseudoprogression; NA, not available; MPM, malignant pleural mesothelioma; SCLC, small cell lung cancer; PS, performance status; NSCLC, non-small cell lung cancer; TGK, tumour growth kinetics. 
the phase III DETERMINE trial. In this study the PD rate according to RECIST v1.1 was slightly lower in the tremelimumab arm (46\%) vs. placebo arm (59\%). Furthermore, OS curves overlapped for the first 3 months from randomization and the ED rate in this timeframe was similar between treatment arms: $21 \%(82 / 382)$ for tremelimumab and 22\% (42/189) for placebo (21).

Nivolumab, an anti-PD1 monoclonal antibody, was tested in pretreated MPM patients enrolled in a single arm phase II Japanese trial (MERIT) (22) and in a Dutch prospective single arm phase II study (23). The PD rates according to RECIST modified for MPM (24) or a combination of RECIST modified for MPM and RECIST modified for ICI (25) were $26 \%$ and $50 \%$, respectively. The ED rates within the first 3 or 4 months of treatment were $18 \%(6 / 34)$ and $9 \%(3 / 34)$ in the Japanese (22) and Dutch (23) trials, respectively. No HPD was reported in both studies, however in the MERIT trial 1 (11\%) out of 9 patients experiencing $\mathrm{PD}$, had an increase of $60 \%$ in the RECIST tumour burden compared to baseline (22).

Pembrolizumab, another anti-PD1 monoclonal antibody, was tested in pretreated MPM patients included in two non-randomized studies, a phase $1 \mathrm{~b}$ (Keynote-028) (26) and a phase II study (27). In both trials, responses were assessed according to RECIST v1.1 and RECIST modified for MPM, respectively and the PD rates were 16\% (26) and $37 \%$ (27). Of note in the phase II trial, 2 (8\%) out of 24 patients with PD experienced an increase of $100 \%$ in the RECIST tumour burden compared to baseline (27). In the phase $1 \mathrm{~b}$ trial, an ED rate in the first 3 months of $16 \%(4 / 25)$ was reported (26).

Pembrolizumab was the only ICI compared to single agent chemotherapy (gemcitabine or vinorelbine) in a randomized phase 3 trial (ETOP 9-15 PROMISE) (28). Although the overall response rate (ORR) was significantly higher with pembrolizumab compared to chemotherapy (22\% vs. 6\%), survival did not differ between treatment arms and the PD rates according to RECIST v1.1 also were similar ( $45 \%$ with pembrolizumab vs. $49 \%$ with single agent chemotherapy). Two patients (one in pembrolizumab arm and one in chemotherapy arm) had an increase of $60 \%$ in the RECIST tumour burden compared to baseline. Of note, in the first 4 months of treatment single agent chemotherapy performed better than pembrolizumab and the ED rate in this timeframe was $24 \%$ in the pembrolizumab arm vs. $18 \%$ in the chemotherapy arm (28).

Avelumab, an anti-PD-L1 monoclonal antibody, was administered in advanced pretreated MPM enrolled in a phase $1 \mathrm{~b}$ trial (JAVELIN). The PD rate according to RECIST $v 1.1$ and the ED rate in the first 3 months of treatment were $34 \%$ and $24 \%$ (13/53). Interestingly, 1 $(5.5 \%)$ out of 18 patients with PD, had a $90 \%$ increase in the RECIST tumour burden compared to baseline (29).

Durvalumab, an anti-PD-L1 monoclonal antibody, has been tested as maintenance treatment after six cycles of chemotherapy in treatment naïve MPM (30). In this single arm phase II study (DREAM trial), PD rates were $15 \%$ and $13 \%$ according to RECIST modified for MPM and RECIST modified for ICI. 2 (28\%) out of 7 patients with PD upon durvalumab had an increase higher than $130 \%$ in the RECIST tumour burden compared to baseline (30). ED rate in the first 3 months of treatment was 7\% (4/54).

Four trials have tested PD-1/PD-L1 inhibitors in combination with anti-CTLA-4 agents in advanced MPM. The first study was a single arm phase II trial (NIBITMESO) in MPM patients (30\% treatment naïve) receiving tremelimumab and durvalumab. The PD rates were of $35 \%$ and $38 \%$ according to immunorelated modified RECIST (31) or RECIST modified for MPM (24), respectively. The ED rate in the first 6 months was $10 \%$ (4/40) (32). INITIATE was a single arm phase II study of nivolumab and ipilimumab, an anti-CTLA-4 monoclonal antibody in pretreated MPM. The PD rate according to RECIST modified for MPM (24) was $32 \%$ and $6 \%(2 / 34)$ of patients died in the first 3 months of treatment (33).

MAPS-2 study was a double arm non-comparative trial testing nivolumab or nivolumab plus ipilimumab in pretreated advanced MPM. ED rates in the first 5 months after randomization were $30 \%(19 / 63)$ and $21 \%(13 / 62)$ in nivolumab and nivolumab plus ipilimumab, respectively. The PD rates according to RECIST modified for MPM (24) were high for both nivolumab $(60 \%)$ and nivolumab plus ipilimumab (48\%) (34). Interestingly 1 patient upon nivolumab and 1 upon nivolumab plus ipilimumab experienced striking PDs with an increase higher than $160 \%$ in the RECIST tumour burden compared to baseline (34). MAPS-2 was the only study were pre-ICI radiological evaluation were collected and used to assess HPD. According to the volumetric criteria previously used in NSCLC, HPD defined as RECIST PD and $>50 \%$ increase in the tumour growth rate (TGR) during ICI compared to TGR before ICI (11) was found in $6(5 \%)$ out of 125 patients ( 4 patients in the nivolumab arm and 2 patients in the nivolumab and ipilimumab arm) and median OS did not differ between HPD and conventional RECIST $\mathrm{PD}$ both in nivolumab and in nivolumab plus ipilimumab 
arms. Considering that in the TGR computation, tumour is considered as a sphere (35) and that mesothelioma rarely harbours spherical features, the TGR definition is difficult to be applied. Tumour growth kinetics (TGK) definition was previously used to assess HPD in head and neck tumours and HPD was defined as a doubling in the RECIST sum of longest diameter (SLD) of target lesions upon ICI compared to pretreatment period (10). According to TGK definition, HPD was reported in 11 (9\%) out of 125 MPM patients in the MAPS-2 trial (7 patients in the nivolumab arm and 4 patients in the nivolumab plus ipilimumab arm). Of note, HPD was significantly associated with negative PD-L1 expression $(<1 \%)$ on tumour cells $(8$ out of 11 HPD patients had negative PD-L1 expression). In addition, patients with conventional disease PD had a better median OS compared to HPD patients according to TGK definition in the whole population [5.5 (95\% CI: 2.6-8.9) vs. 2.6 (95\% CI: 0.8-7.7) months, HR: 0.37 (95\% CI: 0.19 0.75), $\mathrm{P}=0.006$ ] (36).

Finally, at World Lung Cancer Conference 2020, Checkmate 743 phase 3 trial showed promising survival results in favour of nivolumab and ipilimumab combination compared to cisplatin and pemetrexed, in particular in the sarcomatoid subtype. In the overall population and in patients with epithelioid or sarcomatoid histology ED rates were 10\% $(30 / 303), 10 \%(22 / 229)$ and $11 \%(8 / 74)$ respectively in the immunotherapy arm vs. 11\% (34/302), 10\% (23/227) and $15 \%(11 / 75)$ in the chemotherapy arm (37).

If data regarding HPD in MPM from clinical trials are few, even less evidence is available from retrospective cohorts. In the first study of HPD in patients with different tumour types treated with anti-PD-1/PD-L1 agents in phase I trials, only 1 out of 131 patients included had MPM and did not experience HPD according to TGR criteria (8). In another recent study, where HPD was assessed by both TGR and RECIST criteria in a large cohort of cancer patients, 67 out of 270 patients were classified as having other histology, however it was not reported whether MPM were included in that subgroup (18).

Overall, PD (from $15 \%$ up to $60 \%$ ) and ED (from 9\% up to $30 \%$ ) rates reported in clinical trials and specific analyses from MAPS-2 study suggest the occurrence of HPD also in pretreated MPM, both upon PD-1/PD-L1 inhibitors as single agents or in combination with CTLA-4 inhibitors.

The characterization of HPD mechanisms in MPM is an unmet need. In NSCLC patients and patients derived xenografts, an involvement of immune suppressive M2 $\left(\mathrm{CD} 163^{+} \mathrm{CD} 33^{+} \mathrm{PD}-\mathrm{L} 1^{+}\right)$tumour associated macrophages via the Fc portion of anti-PD-1 monoclonal antibody was reported (14). Considering that the immune microenvironment of MPM is dominated by macrophages (38) and that a high $\mathrm{M} 2$ to $\mathrm{CD}^{+} \mathrm{T}$ cells ratio has been associated with poor prognosis in MPM (39), a reprogramming of intratumoral macrophages to M2 upon ICI may occur and be responsible for HPD and ED in MPM.

\section{PD, HPD and ED in SCLC}

In pretreated SCLC patients, nivolumab was compared to topotecan in Checkmate 331 trial (40). Although PD rates were not available, the ED rates in the first 3 months were similar: $26 \%(75 / 284)$ in the nivolumab arm and $22 \%(64 / 285)$ in the topotecan arm. A pooled analysis from pretreated SCLC cohorts receiving single agent pembrolizumab in the phase $1 \mathrm{~b}$ KEYNOTE 028 and in the phase II KEYNOTE 158 studies showed a PD rate of 54\% and an ED rate in the first 3 months of treatment of $23 \%$ (19/83). Of note 1 (2\%) of 45 patients with PD experienced an increase of $100 \%$ in the RECIST tumour burden compared to baseline (41).

Atezolizumab, an anti-PD-L1 monoclonal antibody, was tested in a randomized non-comparative phase II trial (IFCT-1603) versus topotecan or reinduction of initial chemotherapy. PD rates were higher compared to any other trial with $70 \%$ of $\mathrm{PD}$ as best response in the atezolizumab arm compared to $30 \%$ in the chemotherapy arm (42).

In pretreated SCLC patients, PD-1/PD-L1 inhibitors were tested also in combination with anti-CTLA-4 agents. In Checkmate 032 phase 1-2 trial, SCLC patients were randomized to nivolumab or nivolumab and ipilimumab after a failure of at least one line of platinumbased chemotherapy. PD rates were $10 \%$ and $18 \%$ with nivolumab and nivolumab plus ipilimumab respectively. ED rates in the first 3 months were also similar 38\% (56/147) and $37 \%(36 / 96)(43)$.

Nivolumab and nivolumab plus ipilimumab were also compared to placebo as maintenance treatments after platinum-based chemotherapy in extensive stage SCLC (Checkmate 451 trial). PD rates were similar in nivolumab (46\%) and nivolumab plus ipilimumab arm (46\%) while were slightly higher in the placebo arm (57\%). ED rates in the first 3 months from randomization were 13\% (38/280), $17 \%(49 / 279)$ and $14 \%$ (38/275) in nivolumab, nivolumab plus ipilimumab and placebo groups (44).

Six studies tested combination of ICI and platinum-based chemotherapy in treatment naïve SCLC. Ipilimumab was 
tested in combination with platinum-based chemotherapy showing a PD rate of $6 \%$ in the combination arm and $9 \%$ in the chemotherapy arm. The ED rates in the first 4 months from randomization were $6 \%(28 / 478)$ and $5 \%(22 / 476)$ in the ipilimumab plus chemotherapy and chemotherapy alone, respectively (45). In the KEYNOTE 604 phase 3 trial, pembrolizumab in combination with platinum-etoposide was compared to chemotherapy alone. Although a significant survival benefit was observed in the pembrolizumab arm, ED rate in the first 3 months was double in the immunotherapy arm compared to chemotherapy [12\% (27/223) vs. 5\% (12/223)] (46). Similar shapes of survival curves with likely similar ED rates were observed in the ECOG-ACRIN EA5161 phase 2 study comparing platinum-etoposide with nivolumab or placebo in untreated extensive stage SCLC (47).

In the Impower 133 trial, SCLC patients were randomized to first-line platinum-etoposide with atezolizumab or placebo. PD rates and ED rates in the first 3 months were $11 \%$ vs. $9 \%$ and $9 \%(19 / 201)$ vs. $8 \%(16 / 202)$ respectively in the atezolizumab arm compared to placebo (48). Finally, durvalumab was tested in combination with platinumetoposide in the CASPIAN trial. PD rates were $12 \%$ for both arms, ED rates in the first 3 months after randomization were $9 \%(24 / 268)$ in the durvalumab arm vs. $10 \%(27 / 269)$ in the placebo arm (49). The same trial included also an arm of durvalumab plus tremelimumab in combination to platinum-etoposide which showed no median OS improvement compared to platinum-etoposide. In the first 3 months, ED rate was 11\% (30/268), similar to the ED rates in the chemotherapy or durvalumab plus chemotherapy arms (50). Finally, combination of platinumetoposide and pembrolizumab was tested as maintenance therapy after induction with two cycles of platinumetoposide in one EORTC phase 2 study (REACTION), showing improvement in OS with similar ED rate (7\%) in the immunotherapy and in the placebo arm (51).

So far, HPD has not been reported in SCLC, however, in some retrospective series assessing HPD upon ICI it was not specified the histology subtype of lung cancer patients and it's possible that SCLC were included. In Champiat et al. (8) and in Kanjanapan et al. (52), 13 of 131 and 28 of 182 patients respectively had lung cancer with no specified histology subtype. Furthermore, in Champiat et al. no lung cancer patient experienced HPD, in Kanjanapan et al. the rate of HPD in lung cancer subgroup was not reported. Singavi et al. (53) identified 5 patients with HPD according to TGR variation and 1 of them had lung neuroendocrine tumour harbouring FGF3, FGF4 amplification.

Overall, considering the PD (from 6\% to $70 \%$ ) and ED (from $6 \%$ up to $38 \%$ ) rates reported in clinical trials, it is not possible to exclude the occurrence of HPD in SCLC patients particularly upon single agent ICI administered in second or further line.

\section{PD, HPD and ED in thymic malignancies}

Pembrolizumab was tested in a single arm phase II Korean trial including patients with thymoma or thymic carcinoma. PD rates were $0 \%$ and $7 \%$ in thymoma and thymic carcinoma. ED rates in the first 6 months of treatment were $14 \%(1 / 7)$ of thymoma and $23 \%(6 / 26)$ for thymic carcinoma. All patients experiencing PD upon pembrolizumab had a change of RECIST tumour burden from baseline $<40 \%$ (54).

Pembrolizumab was also tested in a single arm phase 2 trial including only thymic carcinoma due to the $71 \%$ grade 3-4 adverse events observed in thymoma patients from the Korean study. PD rate and ED rate in the first 3 months (100 days) were $25 \%$ and $7.5 \%(3 / 40)$. Of note, one of 10 patients experiencing PD had an increase in the RECIST tumour burden from baseline of $140 \%$ (55). Altogether these data suggest that although rare, striking or rapid PDs with deaths may occur in thymic malignancies upon single agent ICI.

\section{PD, HPD and ED in uncommon populations}

The efficacy of ICI in special populations, such as elderly, patients with HIV infection, poor ECOG PS, autoimmune disorders (AID) and uncommon histology is controversial because these categories of patients were excluded or less represented in clinical trials and most of available evidence comes from retrospective or real-world studies.

Regarding elderly NSCLC population, although the age cut-offs used in clinical trials were different, one metanalysis (56) and some studies including patients older than 75 years (57-59) suggested an absence of a significant survival benefit of ICI in this subgroup. On the other hand, a pooled analysis of three trials comparing pembrolizumab to chemotherapy in NSCLC patients (60), a phase II study of nivolumab including $34 \%$ of patients older than 70 years (61) and real-world data (62-65) showed a survival improvement with single agent ICI in the elderly population with no safety concerns. In pretreated NSCLC patients, PD rates upon single agent nivolumab were 
$50 \%$ for both patients younger than 65 years and between 65-75 years (64) and ranged between 13\% (61) and 54\% (64) for NSCLC patients older than 75 years in a phase II trial and in a retrospective study. ED rates in the first 3 months from nivolumab initiation in a phase II trial were $25 \%$ (69/276).

Only one retrospective study assessing HPD in patients treated with single agent PD-1/PD-L1 inhibitors in phase I trials found a statistically significant correlation between HPD and age. In this study, HPD was defined by volumetric criteria as doubling of TGR and HPD rate was $19 \%$ among patients older than 65 years and $5 \%$ among younger patients (8). It's likely that immunological age rather than chronological age impairs ICI efficacy. Specifically, the expansion of low replicative, proinflammatory and oligoclonal senescent T-cells occurring upon persistent antigenic stimulation may negatively affect ICI outcomes (66). To this regard, we recently reported a significant correlation between circulating T-cell immunosenescence and HPD upon ICI in advanced NSCLC patients and all HPD patients had very high rate of circulating senescent $\left(\mathrm{CD} 28^{-} \mathrm{CD} 57^{+} \mathrm{KLRG}^{+}{ }^{+} \mathrm{CD}^{+} \mathrm{T}\right.$-cells (67). Of note, immunosenescence was not associated with age, further suggesting the absence of overlap between chronological and immunological age.

The efficacy of ICI in patients with poor ECOG PS is a debated topic. In fact, baseline ECOG PS $\geq 2$ is associated with worse outcome both in first line setting and in previously treated NSCLC. Interestingly, PD rates were $16 \%$ in 39 pretreated patients included in a phase II trial of nivolumab (Checkmate 171) (61) but raised up to $63 \%$ in 153 treatment naïve PD-L1 $\geq 50 \%$ NSCLC with ECOG PS 2 , with half of them recorded as clinical PD as patients died before any radiological evaluation (68). In this study ED rate in the first 5 months from pembrolizumab initiation was $63 \%$ (97/153). Furthermore, patients with ECOG PS 2 determined by comorbidities had significantly better outcome compared to disease-burden induced PS 2.

In a post-hoc analysis of the OAK trial comparing atezolizumab to docetaxel in pretreated NSCLC patients (69), in a Japanese retrospective study including NSCLC treated with nivolumab (70) and in a Food and Drug Administration (FDA) analysis of different anti-PD-1/PD-L1 agents in NSCLC patients (71) ED rates upon single agent ICI were $5.6 \%, 18.9 \%$ and $9.7 \%$ respectively. Interestingly, in all the three studies ED significantly correlated with worse baseline ECOG PS. Considering that the concept of HPD implies a treatment induced acceleration of the disease during ICI compared to pre-treatment period, it is likely that baseline PS is more a prognostic factor able to predict ED and PD rather than HPD. However, dynamic PS worsening during ICI is a different concept from baseline PS and it was included as a criterium to define HPD in one retrospective study (14). Mechanisms beyond the impairment of immunotherapy efficacy induced by poor ECOG PS remain unclear but may be related to the negative effects of protein catabolism (including rapid clearance of monoclonal antibodies) or to the use of concomitant medications, such as steroids $(72,73)$ or antibiotics $(65,74)$ in poor PS patients.

ICI are being increasingly used in patients with HIV infection and cancer. In a systematic review and metanalysis treatment with ICI was effective and safe, however PD rate was $35 \%$ in NSCLC patients (75). In a phase II trial in HIV patients (DURVAST study), durvalumab was safe and showed a $25 \%$ ORR across different cancer types. PD rate was $44 \%$ and 1 patient had a striking PD with increase in the RECIST tumour burden from baseline of $100 \%$. Among 9 patients with lung cancer (8 NSCLC and 1 SCLC), $4(44 \%)$ had PD as best response to durvalumab (76). In a phase 1 study of pembrolizumab in $\mathrm{HIV}^{+}$cancer patients, PD rate was $27 \%$ and one patient with solid tumour experienced an increase in the RECIST tumour burden from baseline greater than $300 \%$ (19).

Nivolumab and ipilimumab have been administered in $\mathrm{HIV}^{+}$NSCLC patients with ECOG PS 2, or renal/hepatic impairment or untreated brain metastases included in a non-randomized phase 3 trial (Checkmate-871) (77). ORR was $24 \%$ in these special populations, however data on PD and ED rates for single patients' categories were not presented.

In retrospective series including patients with baseline AID treated with ICI (78-80), no correlation was observed between AID and ORR, a no ED or HPD was reported so far.

Finally, the efficacy of ICI in rare NSCLC histotypes is controversial and in a retrospective series including patients with sarcomatoid, enteric, adenosquamous or large cell neuroendocrine tumours a trend towards an higher PD rate $(65 \%$ vs. $47 \%)$ and a decreased OS was reported in rare NSCLC histologies (4.6 months; 95\% CI: 0.03-12.0) compared to common NSCLC (9.2 months; $95 \%$ CI: 7.410.9) (81).

In another retrospective study, including only pretreated NSCLC patients with sarcomatoid histology, although ORR was higher $(40.5 \%)$ compared to historical $15-20 \%$ ORR observed for single agent ICI in further lines. However, PD 
rate was $43 \%(16 / 37), 32 \%(12 / 37)$ of patients had a rapid $\mathrm{PD}$ and half of them $(5 / 37)$ died without a radiological evaluation. Of note three of these rapid PDs had PD-L1 expression on tumour cells of $50 \%, 90 \%$ and $100 \%$ suggesting that PD-L1 in the context of rare histology may lose some of its predictive power (82) in contrast to what observed in other case series (83). The characterization of mechanism of $\mathrm{PD}$ and $\mathrm{ED}$ in patients with rare histotypes is an unmet need. Considering that some molecular alterations such as LKB1 mutation have been associated with HPD in NSCLC patients (13), it is possible that they can be enriched in patients with rare NSCLC histotypes. In this regard, it was recently reported that enteric lung carcinoma showed higher incidence of LKB1 mutations compared to conventional lung adenocarcinoma (84).

\section{PsPD in other thoracic malignancies and uncommon populations}

PsPD has been described as tumour PD followed by radiological response and initially reported in $4.6-9.7 \%$ of melanoma $(85,86)$ and in up to $7 \%$ of renal cell carcinoma (87) treated with ICI.

PsPD is even more uncommon in NSCLC patients, where it occurs in less than $5 \%$ of ICI treated NSCLC patients $(88,89)$. However, no standard definition of PsPD is available so far. In some studies, not only patients with partial or complete response after initial RECIST PD but also patients experiencing prolonged disease stabilization were labelled as pseudoprogressors and the PsPD rate raised at $8-19.5 \%(90,91)$ of ICI treated patients.

In thoracic malignancies other than NSCLC, few data regarding PsPD upon ICI are available.

Three $(9 \%)$ of 34 MPM patients treated with single agent nivolumab in the Dutch trial experienced PsPD defined as partial response after initial RECIST PD (23). Although in the MERIT trial with nivolumab in Japanese MPM patients no PsPD was formally reported, one (3\%) of 34 patients received nivolumab beyond RECIST PD and was still on treatment at 18 months (22).

Two (3\%) of 64 pretreated MPM who received single agent pembrolizumab in a phase II trial showed partial response after initial RECIST PD (27). In the phase $1 \mathrm{~b}$ Keynote-028 trial, no PsPDs were reported, however 1 (4\%) out of 25 patients was treated beyond PD and was still on treatment at 22 months (26). Similarly, in the JAVELIN trial, $1(2 \%)$ out of 53 pretreated MPM, received avelumab beyond RECIST PD and experienced subsequent stable disease (29).
Two (4\%) of 54 MPM receiving single agent durvalumab maintenance in the DREAM trial were pseudoprogressors, having a PD followed by partial response (30). No PsPD were formally reported in trials testing anti-CTLA agents alone or in combination with PD-1/PD-L1 inhibitors (32-34). Interestingly, PsPD in MPM was associated with worsening of clinical condition (23). This finding is peculiar for MPM and was not observed in other tumour types where PsPD is usually characterized by radiological PD and stable clinical PS. It's likely that the immune cell infiltration within the pleura observed during PsPD may affect disease related symptoms much more in MPM compared to other tumour types due to the occurrence of pleural effusions, causing dyspnoea, and the sensitive innervation of the parietal pleura, causing chest pain. The fact that clinical evaluation is not helpful in discriminating between $\mathrm{PsPD}$ and true PD, together with the rarity of PsPD (2-9\%) upon ICI make challenging the use of ICI beyond PD in MPM patients.

Regarding thymic malignancies, although no cases of PsPD have formally been reported, in the Korean study, 1 (3\%) out of 33 patients was treated beyond PD occurred at 30 weeks and was still on pembrolizumab at 66 weeks (54). As far as we are concerned, no PsPD was reported in SCLC patients upon ICI.

Of note, PsPD was not reported for elderly patients, for patients with poor ECOG PS or with rare NSCLC histology. Similarly, in patients with AID no PsPD has been reported, although it's likely that in presence of a more reactive immune system, immune cell infiltration of tumour lesions and subsequent PsPD may more easily occur.

In the phase I trial of pembrolizumab in $\mathrm{HIV}^{+}$cancer patient, one patient experienced an atypical PD with a rapid increase in the tumour burden from baseline followed by a subsequent tumour reduction. This cannot be formerly defined as PsPD, however it suggests that PsPD upon ICI may occur also in the setting of HIV infection (19).

The characterization of mechanisms of PsPD remains an unmet need. Up to now, one study has explored the role of circulating tumour (ct) DNA in melanoma ICI treated patients showing that all 9 patients with PsPD had a favourable ctDNA (undetectable ctDNA or decreased ctDNA by at least 10-fold during treatment) (92). Another potential biomarker of PsPD is decrease in the levels of circulating IL-8. In fact, in two NSCLC patients and one patient with bladder cancer experiencing PsPD upon single agent anti-PD1 treatment, serum IL-8 levels decreased and remained lower compared to baseline (93). Considering 
that IL-8 favours neutrophil infiltration in the tumour microenvironment and the formation of neutrophil extracellular traps (94), it's likely that granulocytic inflammation remains low in patients experiencing PsPD and the initial PD is mainly due to intratumorally infiltration by lymphocytes rather than myeloid cells. Future studies able to characterize mechanisms of PsPD also in other rarer malignancies such as MPM and thymic carcinoma are needed.

\section{Conclusions}

In the immunotherapy era, the identification of patients with rapid PD and deaths upon ICI has found some skepticism and resistance among the scientific community. Nevertheless, HPD upon ICI has been recognized as a phenomenon occurring across different cancer types and evidence regarding rapid PDs and ED is emerging also for other thoracic malignancies such as MPM, SCLC and thymic carcinoma and for elderly patients or patients with HIV infection, poor PS and rare NSCLC histotype. Similarly, PsPD may also occur in other thoracic malignancies (MPM) and in some uncommon populations (i.e., HIV patients), however probably at lower rate compared to HPD. The characterizations of HPD and PsPD mechanisms and the identification of common definition criteria are the next future challenges in this area of cancer research.

\section{Acknowledgments}

Funding: None.

\section{Footnote}

Provenance and Peer Review: This article was commissioned by the Guest editors (Jordi Remon and Benjamin Besse) for the series "Immunotherapy in other thoracic malignancies and uncommon populations" published in Translational Lung Cancer Research. The article has undergone external peer review.

Conflicts of Interest: All authors have completed the ICMJE uniform disclosure form (available at http://dx.doi. org/10.21037/tlcr-20-636). The series "Immunotherapy in other thoracic malignancies and uncommon populations" was commissioned by the editorial office without any funding or sponsorship. Dr. RF reports personal fees from MSD, outside the submitted work. Dr. DS reports personal fees and non-financial support from Astra Zeneca, personal fees and non-financial support from Merck Sharp \& Dohme, personal fees and non-financial support from Bristol Myers Squibb, non-financial support from Roche, outside the submitted work. Dr. CP reports personal fees and other from ROCHE, personal fees and other from BMS, other from MSD, outside the submitted work. Dr. AP reports personal fees from Roche, personal fees from AstraZeneca, personal fees from BMS, outside the submitted work. Dr. MCG reports grants and personal fees from Eli Lilly, personal fees from Boehringer Ingelheim, grants and personal fees from Otsuka Pharma, grants and personal fees from Astra Zeneca, grants and personal fees from Novartis, grants and personal fees from BMS, grants and personal fees from Roche, grants and personal fees from Pfizer, grants and personal fees from Celgene, grants and personal fees from Incyte, personal fees from Inivata, personal fees from Takeda, grants from Tiziana Sciences, grants from Clovis, grants from Merck Serono, grants and personal fees from Bayer, grants and personal fees from MSD, grants and personal fees from GlaxoSmithKline S.p.A., grants and personal fees from Sanofi-Aventis, grants and personal fees from Spectrum Pharmaceutcials, grants and personal fees from Blueprint Medicine, personal fees from Seattle Genetics, personal fees from Daiichi Sankyo, grants from United Therapeutics Corporation, grants from Merck KGaA, personal fees from Janssen, non-financial support from MSD, non-financial support from Eli-Lilly, grants from Turning Point, grants from Ipsen, grants from MedImmune, grants from Exelisis, grants from Array (Pfizer), personal fees from Mirati Therapeutics, outside the submitted work. Dr. GLR reports personal fees from MSD, other from BMS, personal fees from AstraZeneca, other from ROCHE, outside the submitted work. The authors have no other conflicts of interest to declare.

Ethical Statement: The authors are accountable for all aspects of the work in ensuring that questions related to the accuracy or integrity of any part of the work are appropriately investigated and resolved.

Open Access Statement: This is an Open Access article distributed in accordance with the Creative Commons Attribution-NonCommercial-NoDerivs 4.0 International License (CC BY-NC-ND 4.0), which permits the noncommercial replication and distribution of the article with the strict proviso that no changes or edits are made and the 
original work is properly cited (including links to both the formal publication through the relevant DOI and the license). See: https://creativecommons.org/licenses/by-nc-nd/4.0/.

\section{References}

1. Tang J, Shalabi A, Hubbard-Lucey VM. Comprehensive analysis of the clinical immuno-oncology landscape. Ann Oncol 2018;29:84-91.

2. Proto C, Ferrara R, Signorelli D, et al. Choosing wisely first line immunotherapy in non-small cell lung cancer (NSCLC): what to add and what to leave out. Cancer Treat Rev 2019;75:39-51.

3. Prelaj A, Tay R, Ferrara R, et al. Predictive biomarkers of response for immune checkpoint inhibitors in non-smallcell lung cancer. Eur J Cancer 2019;106:144-59.

4. Ferrara R, Pilotto S, Caccese M, et al. Do immune checkpoint inhibitors need new studies methodology? J Thorac Dis 2018;10:S1564-80.

5. Remon J, Vilariño N, Reguart N. Immune checkpoint inhibitors in non-small cell lung cancer (NSCLC): approaches on special subgroups and unresolved burning questions. Cancer Treat Rev 2018;64:21-9.

6. Borcoman E, Kanjanapan Y, Champiat S, et al. Novel patterns of response under immunotherapy. Ann Oncol 2019;30:385-96.

7. Chiou VL, Burotto M. Pseudoprogression and immune-related response in solid tumors. J Clin Oncol 2015;33:3541-3.

8. Champiat S, Ferrara R, Massard C, et al. Hyperprogressive disease: recognizing a novel pattern to improve patient management. Nat Rev Clin Oncol 2018;15:748-62.

9. Kato S, Goodman A, Walavalkar V, et al. Hyperprogressors after immunotherapy: analysis of genomic alterations associated with accelerated growth rate. Clin Cancer Res 2017;23:4242-50.

10. Saâda-Bouzid E, Defaucheux C, Karabajakian A, et al. Hyperprogression during anti-PD-1/PD-L1 therapy in patients with recurrent and/or metastatic head and neck squamous cell carcinoma. Ann Oncol 2017;28:1605-11.

11. Ferrara R, Mezquita L, Texier M, et al. Hyperprogressive disease in patients with advanced non-small cell lung cancer treated with PD-1/PD-L1 inhibitors or with singleagent chemotherapy. JAMA Oncol 2018;4:1543-52.

12. Kim CG, Kim KH, Pyo KH, et al. Hyperprogressive disease during PD-1/PD-L1 blockade in patients with non-small-cell lung cancer. Ann Oncol 2019;30:1104-13.

13. Kim Y, Kim CH, Lee HY, et al. Comprehensive clinical and genetic characterization of hyperprogression based on volumetry in advanced non-small cell lung cancer treated with immune checkpoint inhibitor. J Thorac Oncol 2019;14:1608-18.

14. Lo Russo G, Moro M, Sommariva M, et al. Antibody$\mathrm{Fc} / \mathrm{FcR}$ interaction on macrophages as a mechanism for hyperprogressive disease in non-small cell lung cancer subsequent to PD-1/PD-L1 blockade. Clin Cancer Res 2019;25:989-99.

15. Lo Russo G, Facchinetti F, Tiseo M, et al. Hyperprogressive disease upon immune checkpoint blockade: focus on non-small cell lung cancer. Curr Oncol Rep 2020;22:41.

16. Adashek JJ, Kato S, Ferrara R, et al. Hyperprogression and immune checkpoint inhibitors: hype or progress? Oncologist 2020;25:94-8.

17. Kas B, Talbot H, Ferrara R, et al. Clarification of definitions of hyperprogressive disease during immunotherapy for non-small cell lung cancer. JAMA Oncol 2020;6:1039-46.

18. Matos I, Martin-Liberal J, García-Ruiz A, et al. Capturing hyperprogressive disease with immune-checkpoint inhibitors using RECIST 1.1 Criteria. Clin Cancer Res 2020;26:1846-55.

19. Uldrick TS, Gonçalves PH, Abdul-Hay M, et al. Assessment of the safety of pembrolizumab in patients with HIV and advanced cancer-a phase 1 study. JAMA Oncol 2019;5:1332-9.

20. Ferrara R, Mezquita L, Texier M, et al. Comparison of fast-progression, hyperprogressive disease, and early deaths in advanced non-small-cell lung cancer treated with PD-1/PD-L1 inhibitors or chemotherapy. JCO Precis Oncol 2020;(4):829-40.

21. Maio M, Scherpereel A, Calabrò L, et al. Tremelimumab as second-line or third-line treatment in relapsed malignant mesothelioma (DETERMINE): a multicentre, international, randomised, double-blind, placebocontrolled phase 2b trial. Lancet Oncol 2017;18:1261-73.

22. Okada M, Kijima T, Aoe K, et al. Clinical efficacy and safety of nivolumab: results of a multicenter, open-label, single-arm, Japanese phase II study in malignant pleural mesothelioma (MERIT). Clin Cancer Res 2019;25:5485-92.

23. Quispel-Janssen J, van der Noort V, de Vries JF, et al. Programmed death 1 blockade with nivolumab in patients with recurrent malignant pleural mesothelioma. J Thorac Oncol 2018;13:1569-76.

24. Byrne MJ, Nowak AK. Modified RECIST criteria for assessment of response in malignant pleural mesothelioma. Ann Oncol 2004;15:257-60. 
25. Wolchok JD, Hoos A, O'Day S, et al. Guidelines for the evaluation of immune therapy activity in solid tumors: immune-related response criteria. Clin Cancer Res 2009; 15:7412-20.

26. Alley EW, Lopez J, Santoro A, et al. Clinical safety and activity of pembrolizumab in patients with malignant pleural mesothelioma (KEYNOTE-028): preliminary results from a non-randomised, open-label, phase $1 \mathrm{~b}$ trial. Lancet Oncol 2017;18:623-30.

27. Desai A, Karrison T, Rose B, et al. OA08.03 Phase II Trial of pembrolizumab (NCT02399371) in previously-treated malignant mesothelioma (MM): final analysis. J Thorac Oncol 2018;13:S339.

28. Popat S, Curioni-Fontecedro A, Polydoropoulou V, et al. A multicentre randomized phase III trial comparing pembrolizumab (P) vs single agent chemotherapy (CT) for advanced pre-treated malignant pleural mesothelioma (MPM): results from the European Thoracic Oncology Platform (ETOP 9-15) PROMISE-meso trial. Ann Oncol 2019;30:v931.

29. Hassan R, Thomas A, Nemunaitis JJ, et al. Efficacy and safety of avelumab treatment in patients with advanced unresectable mesothelioma: phase $1 \mathrm{~b}$ results from the JAVELIN solid tumor trial. JAMA Oncol 2019;5:351-7.

30. Nowak A, Kok P, Lesterhuis W, et al. OA08.02 DREAM A phase 2 trial of durvalumab with first line chemotherapy in mesothelioma: final result. J Thorac Oncol 2018;13:S338-9.

31. Nishino M, Giobbie-Hurder A, Gargano M, et al. Developing a common language for tumor response to immunotherapy: immune-related response criteria using unidimensional measurements. Clin Cancer Res 2013;19:3936-43.

32. Calabrò L, Morra A, Giannarelli D, et al. Tremelimumab combined with durvalumab in patients with mesothelioma (NIBIT-MESO-1): an open-label, non-randomised, phase 2 study. The Lancet Respiratory Medicine 2018;6:451-60.

33. Disselhorst MJ, Quispel-Janssen J, Lalezari F, et al. Ipilimumab and nivolumab in the treatment of recurrent malignant pleural mesothelioma (INITIATE): results of a prospective, single-arm, phase 2 trial. Lancet Respir Med 2019;7:260-70.

34. Scherpereel A, Mazieres J, Greillier L, et al. Nivolumab or nivolumab plus ipilimumab in patients with relapsed malignant pleural mesothelioma (IFCT-1501 MAPS2): a multicentre, open-label, randomised, non-comparative, phase 2 trial. Lancet Oncol 2019;20:239-53.

35. Ferté C, Fernandez M, Hollebecque A, et al. Tumor
Growth Rate (TGR) is an early indicator of anti-tumor drug activity in phase I clinical trials. Clin Cancer Res 2014;20:246-52.

36. Zalcman G, Mazieres J, Greillier L, et al. Second/thirdline nivolumab vs nivo plus ipilimumab in malignant pleural mesothelioma: Long-term results of IFCT-1501 MAPS2 phase IIR trial with a focus on hyperprogression (HPD). Ann Oncol 2019;30:v747.

37. Baas P, Scherpereel A, Nowak A, et al. ID:2908 first-line nivolumab + ipilimumab vs chemotherapy in unresectable malignant pleural mesothelioma: CheckMate 743. J Thorac Oncol 2020;15:e42.

38. Lievense LA, Bezemer K, Aerts JGJV, et al. Tumorassociated macrophages in thoracic malignancies. Lung Cancer 2013;80:256-62.

39. Cornelissen R, Lievense LA, Maat AP, et al. Ratio of intratumoral macrophage phenotypes is a prognostic factor in epithelioid malignant pleural mesothelioma. PLoS One 2014;9:e106742.

40. Reck M, Vicente D, Ciuleanu T, Gettinger S, et al. Efficacy and safety of nivolumab (nivo) monotherapy versus chemotherapy (chemo) in recurrent small cell lung cancer (SCLC): Results from CheckMate 331. Ann Oncol 2018;29:x43.

41. Chung HC, Piha-Paul SA, Lopez-Martin J, et al. Pembrolizumab after two or more lines of previous therapy in patients with recurrent or metastatic SCLC: results from the KEYNOTE-028 and KEYNOTE-158 studies. J Thorac Oncol 2020;15:618-27.

42. Pujol JL, Greillier L, Audigier-Valette C, et al. A randomized non-comparative phase II study of antiprogrammed cell death-ligand 1 atezolizumab or chemotherapy as second-line therapy in patients with small cell lung cancer: results from the IFCT-1603 trial. J Thorac Oncol 2019;14:903-13.

43. Ready NE, Ott PA, Hellmann MD, et al. Nivolumab monotherapy and nivolumab plus ipilimumab in recurrent small cell lung cancer: results from the CheckMate 032 randomized cohort. J Thorac Oncol 2020;15:426-35.

44. Owonikoko TK, Kim HR, Govindan R, et al. Nivolumab (nivo) plus ipilimumab (ipi), nivo, or placebo (pbo) as maintenance therapy in patients (pts) with extensive disease small cell lung cancer (ED-SCLC) after first-line (1L) platinum-based chemotherapy (chemo): results from the double-blind, randomized phase III CheckMate 451 study. Ann Oncol 2019;30:ii77.

45. Reck M, Luft A, Szczesna A, et al. Phase III randomized trial of ipilimumab plus etoposide and platinum versus 
placebo plus etoposide and platinum in extensive-stage small-cell lung cancer. J Clin Oncol 2016;34:3740-8.

46. Rudin CM, Awad MM, Navarro A, et al. KEYNOTE-604: Pembrolizumab (pembro) or placebo plus etoposide and platinum (EP) as first-line therapy for extensive-stage (ES) small-cell lung cancer (SCLC). J Clin Oncol 2020;38:9001.

47. Leal T, Wang Y, Dowlati A, et al. Randomized phase II clinical trial of cisplatin/carboplatin and etoposide (CE) alone or in combination with nivolumab as frontline therapy for extensive-stage small cell lung cancer (ES-SCLC): ECOG-ACRIN EA5161. J Clin Oncol 2020;38:9000.

48. Horn L, Mansfield AS, Szczęsna A, et al. First-line atezolizumab plus chemotherapy in extensive-stage smallcell lung cancer. N Engl J Med 2018;379:2220-9.

49. Paz-Ares L, Dvorkin M, Chen Y, et al. Durvalumab plus platinum-etoposide versus platinum-etoposide in firstline treatment of extensive-stage small-cell lung cancer (CASPIAN): a randomised, controlled, open-label, phase 3 trial. Lancet 2019;394:1929-39.

50. Paz-Ares LG, Dvorkin M, Chen Y, et al. Durvalumab \pm tremelimumab + platinum-etoposide in first-line extensivestage SCLC (ES-SCLC): updated results from the phase III CASPIAN study. J Clin Oncol 2020;38:9002.

51. Besse B, Menis J, Bironzo P, et al. LBA85 REACTION: A phase II study of etoposide and cis/carboplatin with or without pembrolizumab in untreated extensive small cell lung cancer. Ann Oncol 2020;31:S1211-2.

52. Kanjanapan Y, Day D, Wang L, et al. Hyperprogressive disease in early-phase immunotherapy trials: Clinical predictors and association with immune-related toxicities. Cancer 2019;125:1341-9.

53. Singavi AK, Harrington AM, Fenske TS. Post-transplant lymphoproliferative disorders. Cancer Treat Res 2015;165:305-27.

54. Cho J, Kim HS, Ku BM, et al. Pembrolizumab for patients with refractory or relapsed thymic epithelial tumor: an open-label phase II trial. J Clin Oncol 2019;37:2162-70.

55. Giaccone G, Kim C, Thompson J, et al. Pembrolizumab in patients with thymic carcinoma: a single-arm, singlecentre, phase 2 study. Lancet Oncol 2018;19:347-55.

56. Landre T, Taleb C, Nicolas $\mathrm{P}$, et al. Is there a clinical benefit of anti-PD-1 in patients older than 75 years with previously treated solid tumour? J Clin Oncol 2016;34:3070.

57. Brahmer J, Reckamp KL, Baas P, et al. Nivolumab versus docetaxel in advanced squamous-cell non-small-cell lung cancer. N Engl J Med 2015;373:123-35.
58. Borghaei H, Paz-Ares L, Horn L, et al. Nivolumab versus docetaxel in advanced nonsquamous non-small-cell lung cancer. N Engl J Med 2015;373:1627-39.

59. Rittmeyer A, Barlesi F, Waterkamp D, et al. Atezolizumab versus docetaxel in patients with previously treated non-small-cell lung cancer (OAK): a phase 3, openlabel, multicentre randomised controlled trial. Lancet 2017;389:255-65.

60. Nosaki K, Hosomi Y, Saka H, et al. 103O_PRSafety and efficacy of pembrolizumab (Pembro) monotherapy in elderly patients (Pts) with PD-L1-positive advanced NSCLC: Pooled analysis from KEYNOTE-010, -024, and -042. Lung Cancer 2019;135:188-95.

61. Felip E, Ardizzoni A, Ciuleanu T, et al. CheckMate 171: A phase 2 trial of nivolumab in patients with previously treated advanced squamous non-small cell lung cancer, including ECOG PS 2 and elderly populations. Eur J Cancer 2020;127:160-72.

62. Spigel D, Schwartzberg L, Waterhouse D, et al. P3.02c-026 Is nivolumab safe and effective in elderly and PS2 patients with non-small cell lung cancer (NSCLC)? Results of CheckMate 153. J Thorac Oncol 2017;12:S1287-8.

63. Bagley S, Kothari S, Aggarwal C, et al. P3.02c-028 Outcomes of nivolumab in elderly patients (pts) with nonsmall cell lung cancer (NSCLC): topic: IT. J Thorac Oncol 2017;12:S1289.

64. Grossi F, Crinò L, Logroscino A, et al. Use of nivolumab in elderly patients with advanced squamous non-small-cell lung cancer: results from the Italian cohort of an expanded access programme. Eur J Cancer 2018;100:126-34.

65. Galli G, De Toma A, Pagani F, et al. Efficacy and safety of immunotherapy in elderly patients with non-small cell lung cancer. Lung Cancer 2019;137:38-42.

66. Ferrara R, Mezquita L, Auclin E, et al. Immunosenescence and immunecheckpoint inhibitors in non-small cell lung cancer patients: does age really matter? Cancer Treat Rev 2017;60:60-8

67. Ferrara R, Naigeon M, Auclin E, et al. Circulating T-cell immunosenescence in advanced non-small cell lung cancer patients treated with single agent PD-1/PD-L1 inhibitors or platinum-based chemotherapy. Clin Cancer Res 2021;27:492-503.

68. Facchinetti F, Mazzaschi G, Barbieri F, et al. First-line pembrolizumab in advanced non-small cell lung cancer patients with poor performance status. Eur J Cancer 2020;130:155-67.

69. Gandara DR, Reck M, Morris S, et al. Fast progression 
in patients treated with a checkpoint inhibitor (cpi) vs chemotherapy in OAK, a phase III trial of atezolizumab (atezo) vs docetaxel (doc) in 2L+ NSCLC. Ann Oncol 2018;29:x39.

70. Inoue T, Tamiya M, Tamiya A, et al. Analysis of early death in Japanese patients with advanced non-small-cell lung cancer treated with nivolumab. Clin Lung Cancer 2018;19:e171-6.

71. Mulkey F, By K, Theoret MR, et al. Analysis of early mortality in randomized clinical trials evaluating antiPD-1/PD-L1 antibodies: a systematic analysis by the United States Food and Drug Administration (FDA). J Clin Oncol 2019;37:2516.

72. Fucà G, Galli G, Poggi M, et al. Modulation of peripheral blood immune cells by early use of steroids and its association with clinical outcomes in patients with metastatic non-small cell lung cancer treated with immune checkpoint inhibitors. ESMO Open 2019;4:e000457.

73. Arbour KC, Mezquita L, Long N, et al. Impact of baseline steroids on efficacy of programmed cell death-1 and programmed death-ligand 1 blockade in patients with nonsmall-cell lung cancer. J Clin Oncol 2018;36:2872-8.

74. Derosa L, Hellmann MD, Spaziano M, et al. Negative association of antibiotics on clinical activity of immune checkpoint inhibitors in patients with advanced renal cell and non-small-cell lung cancer. Ann Oncol 2018;29:1437-44.

75. Cook MR, Kim C. Safety and efficacy of immune checkpoint inhibitor therapy in patients with HIV infection and advanced-stage cancer: a systematic review. JAMA Oncol 2019;5:1049-54.

76. Gonzalez-Cao M, Morán T, Dalmau J, et al. Assessment of the feasibility and safety of durvalumab for treatment of solid tumors in patients with HIV-1 infection: the phase 2 DURVAST study. JAMA Oncol 2020;6:1063-7.

77. Barlesi F, Audigier-Valette C, Felip E, et al. OA04.02 CheckMate 817: first-line nivolumab + ipilimumab in patients with ECOG PS 2 and other special populations with advanced NSCLC. J Thorac Oncol 2019;14:S214-5.

78. Leonardi GC, Gainor JF, Altan M, et al. Safety of programmed death-1 pathway inhibitors among patients with non-small-cell lung cancer and preexisting autoimmune disorders. J Clin Oncol 2018;36:1905-12.

79. Cortellini A, Buti S, Santini D, et al. Clinical outcomes of patients with advanced cancer and pre-existing autoimmune diseases treated with anti-programmed death-1 immunotherapy: a real-world transverse study. Oncologist 2019;24:e327-37.

80. Danlos FX, Voisin AL, Dyevre V, et al. Safety and efficacy of anti-programmed death 1 antibodies in patients with cancer and pre-existing autoimmune or inflammatory disease. Eur J Cancer 2018;91:21-9.

81. Signorelli D, Ferrara R, Proto C, et al. Immunecheckpoints inhibitors in metastatic non small cell lung cancer with rare histology. J Clin Oncol 2019;37:9106.

82. Domblides C, Leroy K, Monnet I, et al. Efficacy of immune checkpoint inhibitors in lung sarcomatoid carcinoma. J Thorac Oncol 2020;15:860-6.

83. Babacan NA, Pina IB, Signorelli D, et al. Relationship between programmed death receptor-ligand 1 expression and response to checkpoint inhibitor immunotherapy in pulmonary sarcomatoid carcinoma: a pooled analysis. Clin Lung Cancer 2020;21:e456-63.

84. Galli G, Imbimbo M, Busico A, et al. P2.09-05 Clinical and biological characterization of lung enteric adenocarcinoma. J Thorac Oncol 2019;14:S770.

85. Hodi FS, Hwu WJ, Kefford R, et al. Evaluation of immune-related response criteria and RECIST v1.1 in patients with advanced melanoma treated with pembrolizumab. J Clin Oncol 2016;34:1510-7.

86. Long GV, Weber JS, Larkin J, et al. Nivolumab for patients with advanced melanoma treated beyond progression: analysis of 2 phase 3 clinical trials. JAMA Oncol 2017;3:1511-9.

87. Escudier B, Motzer RJ, Sharma P, et al. Treatment beyond progression in patients with advanced renal cell carcinoma treated with nivolumab in CheckMate 025. Eur Urol 2017;72:368-76.

88. Ferrara R, Caramella C, Besse B, et al. Pseudoprogression in non-small cell lung cancer upon immunotherapy: few drops in the ocean? J Thorac Oncol 2019;14:328-31.

89. Tazdait M, Mezquita L, Lahmar J, et al. Patterns of responses in metastatic NSCLC during PD-1 or PDL-1 inhibitor therapy: Comparison of RECIST 1.1, irRECIST and iRECIST criteria. Eur J Cancer 2018;88:38-47.

90. Rizvi NA, Mazières J, Planchard D, et al. Activity and safety of nivolumab, an anti-PD-1 immune checkpoint inhibitor, for patients with advanced, refractory squamous non-small-cell lung cancer (CheckMate 063): a phase 2, single-arm trial. Lancet Oncol 2015;16:257-65.

91. Gandara DR, von Pawel J, Mazieres J, et al. Atezolizumab treatment beyond progression in advanced NSCLC: results from the randomized, phase III OAK study. J Thorac Oncol 2018;13:1906-18.

92. Lee JH, Long GV, Menzies AM, et al. Association between circulating tumor DNA and pseudoprogression in patients with metastatic melanoma treated with anti-programmed 
cell death 1 antibodies. JAMA Oncol 2018;4:717-21.

93. Sanmamed MF, Perez-Gracia JL, Schalper KA, et al. Changes in serum interleukin-8 (IL-8) levels reflect and predict response to anti-PD-1 treatment in melanoma and non-small-cell lung cancer patients. Ann Oncol

Cite this article as: Ferrara R, Signorelli D, Proto C, Prelaj A, Garassino MC, Lo Russo G. Novel patterns of progression upon immunotherapy in other thoracic malignancies and uncommon populations. Transl Lung Cancer Res 2021;10(6):2955-2969. doi: 10.21037/tlcr-20-636
2017;28:1988-95.

94. Nie M, Yang L, Bi X, et al. Neutrophil extracellular traps induced by IL8 promote diffuse large B-cell lymphoma progression via the TLR9 signaling. Clin Cancer Res 2019;25:1867-79. 